\title{
Dynamical Behaviors of Impulsive Stochastic Reaction-Diffusion Neural Networks with Mixed Time Delays
}

\author{
Weiyuan Zhang, ${ }^{1,2}$ Junmin $^{2} i_{,}{ }^{1}$ and Minglai Chen ${ }^{1}$ \\ ${ }^{1}$ School of Science, Xidian University, Xi'an 710071, China \\ ${ }^{2}$ Institute of Math and Applied Math, Xianyang Normal University, Xianyang 712000, China \\ Correspondence should be addressed to Junmin Li, jmli@mail.xidian.edu.cn
}

Received 17 April 2012; Accepted 16 June 2012

Academic Editor: Sabri Arik

Copyright (C) 2012 Weiyuan Zhang et al. This is an open access article distributed under the Creative Commons Attribution License, which permits unrestricted use, distribution, and reproduction in any medium, provided the original work is properly cited.

\begin{abstract}
We discuss the dynamical behaviors of impulsive stochastic reaction-diffusion neural networks (ISRDNNs) with mixed time delays. By using a well-known $L$-operator differential inequality with mixed time delays and combining with the Lyapunov-Krasovkii functional approach, as well as linear matrix inequality (LMI) technique, some novel sufficient conditions are derived to ensure the existence, uniqueness, and global exponential stability of the periodic solutions for ISRDNNs with mixed time delays in the mean square sense. The obtained sufficient conditions depend on the reaction-diffusion terms. The results of this paper are new and improve some of the previously known results. The proposed model is quite general since many factors such as noise perturbations, impulsive phenomena, and mixed time delays are considered. Finally, two numerical examples are provided to verify the usefulness of the obtained results.
\end{abstract}

\section{Introduction}

In recent years, neural networks (NNs) with time delays have received considerable attention due to their extensive applications in solving some optimization problems, associative memory, classification of patterns, and other areas. In implementation of NNs, however, time delays are unavoidably encountered. It has been found that the existence of time delays may lead to instability and oscillation in a neural network. Therefore, the analysis of the dynamical behaviors such as stability, periodic oscillation, and chaotic behavior are necessary work for practical design of delayed NNs [1-12]. Zheng and Chen [1] studied the exponential stability for delayed periodic dynamical systems. In [2], the global exponential stability and periodicity of a class of recurrent NNs with time delays are addressed by using Lyapunov functional method and inequality techniques. In the factual operations, however, the diffusion phenomena could not be ignored in NNs when electrons are moving in asymmetric electromagnetic 
fields. So we must consider that the activations vary in space as well as in time. The NNs with diffusion terms can commonly be expressed by partial differential equations [13-33]. The authors in $[13,19,20]$ have dealt with obtaining sufficient conditions for the global exponential stability and periodicity of delayed reaction-diffusion neural networks (RDNNs).

As is well known, besides delays and diffusion effects, impulsive phenomena can be found in a wide variety of evolutionary process, particularly in some biological systems such as biological NNs and bursting rhythm models in pathology, as well as optimal control models in economics, frequency-modulated signal processing systems, and flying object motions, in which many sudden and sharp changes occur instantaneously, in the form of impulse. For example, in implementation of electronic networks, the state of the networks is subject to instantaneous perturbations and experiences abrupt change at certain instants, which may be caused by switching phenomenon, frequency change, or other sudden noise, that is, it exhibits impulsive effects. As artificial electronic system, neural networks are often subject to impulsive perturbations that in turn affect dynamical behaviors of the systems $[17,18,25-27]$. In [17, 26, 27], the global exponential stability for the equilibrium point of impulsive RDNNs with delays was investigated.

However, the models studied in the above mentioned papers have been largely restricted to deterministic RDNNs. In the real world, a real system is usually affected by external perturbations which in many cases are of great uncertainty and hence may be treated as random. As pointed out by Haykin [34] that in real nervous systems synaptic transmission is a noisy process brought on by random fluctuations from the release of neurotransmitters and other probabilistic causes. Hence, it is of significant importance to study stochastic effects for the neural networks. In recent years, the dynamic behavior of stochastic NNs, especially the stability of stochastic NNs, has become a hot study topic. Very recently, several kinds of NNs with delays and stochastic effects have been investigated [22, 28-30]. Lv et al. [22] and $\mathrm{Xu}$ et al. [29] have obtained some criteria to guarantee the almost sure exponential stability and mean square exponential stability of an equilibrium solution for RDNNs with continuously distributed delays and stochastic influence, respectively. It is noticed that the authors do not take impulsive phenomena and diffusion effects into account on the dynamic behaviors of RDNNs.

It is well known that not only diffusion effects and delays cannot be avoided but also the existence of impulsive and stochastic effects is extensive in the NNs. Moreover, the interconnection weights $b_{i j}, \widetilde{b}_{i j}, \bar{b}_{i j}$, self-inhibition $a_{i}$ and inputs $J_{i}$ in the NNs may be variable with time, often periodically. Therefore, it is necessary to consider impulsive and stochastic effects to the stability of RDNNs with mixed time delays and their periodic limits. Unfortunately, to the best of our knowledge, the existence and global exponential stability of periodic solutions have been seldom considered for ISRDNNs with variable coefficients and mixed time delays. Due to the simultaneous presence of impulsive stochastic effects, reaction-diffusion phenomena, periodicity, variable coefficients, and mixed time delays, the dynamical behaviors become much more complex and therefore pose significant difficulties in the analysis.

Based on the above discussions, in this paper, we aim to challenge the analysis problem on dynamical behaviors of ISRDNNs with mixed time delays. By applying a well-known $L-$ operator differential inequality with mixed time delays and combining with the LyapunovKrasovkii functional approach, as well as linear matrix inequality (LMI) technique, we have derived some easy-to-test sufficient conditions for the existence and exponential stability of the periodic solutions for ISRDNNs with variable coefficients and mixed time delays. The obtained criteria depend on the reaction-diffusion terms. The results of this paper are new and they complement previously known results. Furthermore, we do not need the 
differentiability of the time-varying delays. Two examples are employed to demonstrate the effectiveness of the obtained results that are less restrictive than recently known criteria.

Notation. Throughout this paper, the following notations will be used. $\mathbb{R}^{n}$ and $\mathbb{R}^{n \times n}$ denote the $n$-dimensional Euclidean real space equipped with the norm $|\cdot|$ and the set of all $n \times n$ real matrices, respectively. Trace $(\cdot)$ denotes the trace of the corresponding matrix and $I$ denotes the identity matrix with appropriate dimensions. For square matrices $A$ and $B$, the notation $A>(\geq,<, \leq) B$ denotes $A-B$ is positive-definite (positive-semidefinite, negative, negative-semidefinite) matrix. $L$ denotes the well-known $L$-operator given by the Ito formula. Let $w(t)=\left(w_{1}(t), \ldots, w_{n}(t)\right)^{T}$ is an $n$-dimensional standard Brownian motion defined on a complete probability space $\left(\Omega, F,\left\{F_{t}\right\}_{t \geq 0}, P\right)$ with a natural filtration $\left\{F_{t}\right\}_{t \geq 0}$ generated by $\{w(s): 0 \leq s \leq t\}$. $E(\cdot)$ stands for the mathematical expectation operator. $Z^{+}$is the set of nonnegative integral numbers.

$P \mathbb{C}\left[(-\infty, 0] \times \Omega, \mathbb{R}^{n}\right]=\left\{\psi:(-\infty, 0] \times \Omega \rightarrow \mathbb{R}^{n} \mid \psi\left(s^{+}, x\right)=\psi(s, x)\right.$ for $s \in(-\infty, 0]$, $\psi\left(s^{-}, x\right)$ exists for $s \in(-\infty, 0], \psi\left(s^{-}, x\right)=\psi(s, x)$ for all but at most countable points $s \in$ $(-\infty, 0]\}$, where $\psi\left(t^{-}, x\right)$ and $\psi\left(t^{+}, x\right)$ denote the left-hand limit and the right-hand limit of $\psi(t, x)$ at time $t$, respectively. Especially, let $P \mathbb{C}=P \mathbb{C}\left[(-\infty, 0] \times \Omega, \mathbb{R}^{n}\right]$. For $\psi \in P \mathbb{C}$, we always assume that $\psi$ is bounded and introduce the norm $\|\psi\|=\sup _{-\infty \leq s \leq 0}\left(\sum_{i=1}^{n} \psi_{i}^{2}(s)\right)^{1 / 2}$.

Let $P \mathbb{C}_{F_{0}}^{b}\left[(-\infty, 0] \times \Omega, \mathbb{R}^{n}\right]$ denote the family of all bounded $F_{0}$-measurable, $P \mathbb{C}[(-\infty$, $0] \times \Omega, \mathbb{R}^{n}$ ]-valued random variables $\psi$, such that $\|\psi\|_{\tau}=\sup _{-\infty \leq s \leq 0} E|\psi(s)|^{2}<\infty$. Especially, let $P \mathbb{C}_{F_{0}}^{b}=P \mathbb{C}_{F_{0}}^{b}\left[(-\infty, 0] \times \Omega, \mathbb{R}^{n}\right]$. Let $u=\left(u_{1}, \ldots, u_{n}\right)^{T} \in \mathbb{R}^{n}$ and $L^{2}(\Omega)$ is the space of scalar value Lebesgue measurable functions on $\Omega$ which is a Banach space for the $L_{2}$-norm:

$$
\|u\|_{2}=\left(\int_{\Omega}|u(x)|^{2} d x\right)^{1 / 2}, \quad u \in L^{2}(\Omega)
$$

where $|\cdot|$ is Euclid norm of a vector $u \in \mathbb{R}^{n}$.

\section{Model Description and Preliminaries}

Consider the following ISRDNNs with mixed time delays system:

$$
\begin{aligned}
& d u_{i}(t, x)=\sum_{l=1}^{m} \frac{\partial}{\partial x_{l}}\left(D_{i l} \frac{\partial u_{i}(t, x)}{\partial x_{l}}\right) d t \\
& +\left[-a_{i}(t) u_{i}(t, x)+\sum_{j=1}^{n} b_{i j}(t) f_{j}\left(u_{j}(t, x)\right)+\sum_{j=1}^{n} \tilde{b}_{i j}(t) \tilde{f}_{j}\left(u_{j}(t-\tau(t), x)\right)\right. \\
& \left.+\sum_{j=1}^{n} \bar{b}_{i j}(t) \int_{-\infty}^{t} k_{i j}(t-s) \bar{f}_{j}\left(u_{j}(s, x)\right) d s+J_{i}(t)\right] d t \\
& +\sum_{j=1}^{n} \sigma_{i j}(t, x, u(t, x), u(t-\tau(t), x)) d w_{j}(t), \quad t \geq 0, t \neq t_{k}, x \in \Omega, k \in Z^{+}, \\
& u_{i}(t, x)=u_{i}\left(t^{-}, x\right)-\theta_{i k} u_{i}\left(t^{-}, x\right), \quad t=t_{k}, x \in \Omega, k \in Z^{+},
\end{aligned}
$$

where $i \in N=\{1,2, \ldots, n\}, n \geq 2$, corresponds to the number of units in an NN; the time sequence $t_{k}$ is called impulsive moment and satisfies $0<t_{0}<t_{1}<\cdots<t_{k}<t_{k+1}<\cdots$, 
$\lim _{k \rightarrow \infty} t_{k+1}=\infty ; \theta_{i k}$ are some real constants; $x=\left(x_{1}, \ldots, x_{m}\right)^{T} \in \Omega, \Omega$ is a compact set with smooth boundary $\partial \Omega$ and mes $\Omega>0$ in space $\mathbb{R}^{m}$, where mes $\Omega$ is the measure of the set $\Omega ; u_{i}(t, x)$ represents the state of the $i$ th neuron at time $t$ and in space $x ; b_{i j}(t), \widetilde{b}_{i j}(t)$, and $\bar{b}_{i j}(t)$ denote the strength of the $j$ th neuron on the $i$ th neuron, respectively; $f_{j}, \tilde{f}_{j}$, and $\bar{f}_{j}$ denote the activation functions of the $j$ th neuron at time $t$ and in space $x$; $J_{i}$ denotes the external inputs on the $i$ th neurons; $a_{i}(t)$ is the rate with which the $i$ th unit will reset its potential to the resting state in isolation when disconnected from the network and external inputs at time $t$ and in space $x ; \tau(t)$ represents the transmission delay with $0 \leq \tau(t) \leq \tau, \tau$ is a constant; smooth functions $D_{i l}>0(i=1,2, \ldots, n, l=1,2, \ldots, m)$ stand for the transmission diffusion operators along the $i$ th neuron; the delay kernel $k_{i j}(\cdot)$ is the real value nonnegative continuous function defined on $(0,+\infty) ; u_{i}\left(t^{-}, x\right)$ and $u_{i}\left(t^{+}, x\right)$ denote the left-hand limit and the right-hand limit of $u_{i}(t, x)$ at time $t$, respectively. We assume $u_{i}\left(t_{k}, x\right)=u_{i}\left(t_{k}^{+}, x\right)$.

$\sigma_{i j}(t, x, u(t, x), u(t-\tau(t), x))(i, j=1,2, \ldots, n)$ denotes the weight function of random perturbation.

The boundary conditions and initial conditions are given by

$$
\begin{gathered}
u_{i}(t, x)=0, \quad(t, x) \in[0,+\infty) \times \partial \Omega, \\
u_{i}\left(t_{0}+s, x\right)=\psi_{i}(s, x), \quad(s, x) \in(-\infty, 0] \times \Omega,
\end{gathered}
$$

where $\psi=\left(\psi_{1}, \ldots, \psi_{n}\right)^{T} \in P \mathbb{C}_{F_{0}}^{b}$.

In fact, some famous NNs models became a special case of system (2.1). For example, when $\sigma_{i j}=0, i, j \in N$, the special case of system (2.1) is the model which has been investigated $[25,27,32]$. When $\theta_{i k}=0, i=1,2, \ldots, n, k \in Z^{+}$, then system (2.1) becomes stochastic RDNNs with mixed delays, which has been considered in [22, 29]. If $\theta_{i k}=0$ and $\sigma_{i j}=0, i, j \in N, k \in Z^{+}$, system (2.1) reduces to the deterministic system with mixed time delays:

$$
\begin{aligned}
\frac{d u_{i}(t)}{d t}= & \sum_{l=1}^{m} \frac{\partial}{\partial x_{l}}\left(D_{i l} \frac{\partial u_{i}(t)}{\partial x_{l}}\right)-a_{i}(t) u_{i}(t)+\sum_{j=1}^{n} b_{i j}(t) f_{j}\left(u_{j}(t, x)\right) \\
& +\sum_{j=1}^{n} \tilde{b}_{i j}(t) \tilde{f}_{j}\left(u_{j}(t-\tau(t), x)\right)+\sum_{j=1}^{n} \bar{b}_{i j}(t) \int_{-\infty}^{t} k_{i j}(t-s) \bar{f}_{j}\left(u_{j}(s, x)\right) d s+J_{i},
\end{aligned}
$$

the dynamical behaviors of the special case for model (2.3) have been discussed by many authors $[19,20]$. Therefore, the model $(2.1)$ is new and more general than those discussed in the previous literature.

Throughout this paper, we assume that the following conditions are made.

(A1) Suppose that $a_{i}(t)>0, b_{i j}(t), \tilde{b}_{i j}(t), \bar{b}_{i j}(t), \tau(t) \geq 0$ and $J_{i}(t)$ are all continuously periodic functions defined on $[0,+\infty)$ with common period $\omega>0$. Moreover,

$\widehat{a}_{i}=\min _{t \in[0, \omega]}\left\{a_{i}(t)\right\}, \quad \widehat{b}_{i j}=\max _{t \in[0, \omega]}\left\{\left|b_{i j}(t)\right|\right\}, \quad \widehat{\widetilde{b}}_{i j}=\max _{t \in[0, \omega]}\left\{\left|\widetilde{b}_{i j}(t)\right|\right\}, \quad \widehat{\bar{b}}_{i j}=\max _{t \in[0, \omega]}\left\{\left|\bar{b}_{i j}(t)\right|\right\}$,

$$
i, j \in N \text {. }
$$


(A2) There exist positive diagonal matrices $L^{f}=\operatorname{diag}\left(L_{1}^{f}, \ldots, L_{n}^{f}\right), L^{\tilde{f}}=\operatorname{diag}\left(L_{1}^{\tilde{f}}, \ldots, L_{n}^{\tilde{f}}\right)$, $L^{\bar{f}}=\operatorname{diag}\left(L_{1}^{\bar{f}}, \ldots, L_{n}^{\bar{f}}\right)$, such that for all $\eta_{1}, \eta_{2} \in \mathbb{R}$

$$
\begin{gathered}
\left|f_{j}\left(\eta_{1}\right)-f_{j}\left(\eta_{2}\right)\right| \leq L_{j}^{f}\left|\eta_{1}-\eta_{2}\right|, \\
\left|\tilde{f}_{j}\left(\eta_{1}\right)-\tilde{f}_{j}\left(\eta_{2}\right)\right| \leq L_{j}^{\tilde{f}}\left|\eta_{1}-\eta_{2}\right|, \\
\left|\bar{f}_{j}\left(\eta_{1}\right)-\bar{f}_{j}\left(\eta_{2}\right)\right| \leq L_{j}^{\bar{f}}\left|\eta_{1}-\eta_{2}\right|, \quad j=1,2, \ldots, n .
\end{gathered}
$$

(A3) The delay kernel $k_{i j}(\cdot):[0,+\infty) \rightarrow[0,+\infty),(i, j \in N)$ are real-valued nonnegative continuous functions that satisfy the following conditions:

(i) $\int_{0}^{+\infty} k_{i j}(s) d s=1$,

(ii) $k_{i j}(s) \leq \kappa(s)$ for all $s \in[0,+\infty)$, in which $\kappa(s):[0,+\infty) \rightarrow R^{+}$is continuous and integral and satisfies $\int_{0}^{+\infty} \kappa(s) e^{\eta s} d s<+\infty$, where the constant $\eta$ denotes some positive number.

(A4) For $\omega>0$, there exists $q \in Z^{+}$such that $t_{k}+\omega=t_{k+q}$ and $\theta_{i k}+\omega=\theta_{i(k+q)}, k \in Z^{+}, i \in$ $N$.

(A5) There exist nonnegative constants $\delta_{i}$ and $\gamma_{i}$ such that

$$
\left(\sigma_{i}\left(t, x, \xi_{i}^{\prime}, \varsigma_{i}^{\prime}\right)-\sigma_{i}\left(t, x, \xi_{i}, \varsigma_{i}\right)\right)\left(\sigma_{i}\left(t, x, \xi_{i}^{\prime}, \varsigma_{i}^{\prime}\right)-\sigma_{i}\left(t, x, \xi_{i}, \varsigma_{i}\right)\right)^{T} \leq \delta_{i}\left|\xi_{i}^{\prime}-\xi_{i}\right|^{2}+\gamma_{i}\left|\varsigma_{i}^{\prime}-\varsigma_{i}\right|^{2},
$$

for all $\xi_{i}, \varsigma_{i}, \xi_{i}^{\prime}, \varsigma_{i}^{\prime} \in \mathbb{R}, \sigma_{i}(t, x, \xi, \varsigma)=\left(\sigma_{i 1}(t, x, \xi, \varsigma), \ldots, \sigma_{i n}(t, x, \xi, \varsigma)\right)$ is the $i$ th row vector of $\sigma(t, x, \xi, \varsigma), i \in N$.

For convenience, $u_{i}(t, x), \psi_{i}(s, x)$ are denoted as $u_{i}(t)$ or $u_{i}, \psi_{i}(s)$ or $\psi_{i}$, respectively, if no confusion should occur.

Definition 2.1. An equilibrium point $u^{*}=\left(u_{1}^{*}, u_{2}^{*}, \ldots, u_{n}^{*}\right)$ of system (2.1)-(2.2) is said to be globally exponentially stable in the mean square sense if there exist positive constants $\varepsilon$ and $M \geq 1$ such that

$$
E\left\|u(t, x)-u^{*}\right\|_{2} \leq M\left\|\psi-u^{*}\right\|_{\tau} e^{-\varepsilon\left(t-t_{0}\right)}, \quad t \geq t_{0} \geq 0
$$

Definition 2.2. The system (2.1)-(2.2) is said to be globally exponentially periodic in the mean square sense if (i) there exist one $\omega$-periodic solutions; (ii) all other solutions of the system converge exponentially to it in the mean square sense as $t \rightarrow+\infty$.

Lemma 2.3 (see [24]). Let $\Omega$ be a cube $\left|x_{i}\right|<d_{l}(l=1, \ldots, m)$ and let $h(x)$ be a real-valued function belonging to $C^{1}(\Omega)$ which vanish on the boundary $\partial \Omega$ of $\Omega$, that is, $\left.h(x)\right|_{\partial \Omega}=0$. Then

$$
\int_{\Omega} h^{2}(x) d x \leq d_{l}^{2} \int_{\Omega}\left|\frac{\partial h}{\partial x_{i}}\right| d x
$$


Remark 2.4. The boundary conditions of the investigated RDNNs in [22, 24, 26-28, 35] are all the Neumann boundary conditions. The obtained global exponential stability criteria are independent of diffusion term. In other words, these criteria are same whether the diffusion terms exist or not. However, it is also common to consider the diffusion effects in biological systems (such as immigration [36]). In this paper, we investigate dynamical behaviors of ISRDNNs with Dirichlet boundary conditions and mixed delays. The obtained criteria depend on the reaction-diffusion terms. The Lemma 2.3 plays a key role in the reported criteria which are dependent of diffusion terms.

Lemma 2.5 (see [4]). Let $p, q, r$, and $\beta_{k},\left(k \in Z^{+}\right)$be nonnegative constants, and function $V(x) \in$ $P \mathbb{C}^{2}\left(\mathbb{R}^{n}, \mathbb{R}^{+}\right), L V$ associated with system (2.1), satisfy the following inequalities:

$$
\begin{gathered}
L V(x(t)) \leq-p V(x(t))+q \sup _{t-\tau \leq s \leq t} V(x(s))+r \int_{0}^{+\infty} \kappa(s) V(x(t-s)) d s, \quad t \neq t_{k}, t \geq 0, \\
V\left(x\left(t_{k}\right)\right) \leq \beta_{k} V\left(x\left(t_{k}^{-}\right)\right), \quad k \in Z^{+},
\end{gathered}
$$

where $\kappa(s)$ is the same as (A3). Assume that

(i) $p>q+r \int_{0}^{+\infty} \kappa(s) d s$;

(ii) there exist constants $M>0, \alpha>0$ such that

$$
\prod_{k=1}^{n} \max \left\{1, \beta_{k}\right\} \leq M e^{\alpha t_{n}}, \quad n \in Z^{+} .
$$

Then

$$
E V(x(t)) \leq M E V_{0} e^{-(\lambda-\alpha) t}, \quad t \geq t_{0}
$$

where $E V_{0}=\sup _{-\infty<s \leq 0} E V(x(s)), \lambda \in(0, \eta)$ satisfies $\lambda<p-q e^{\lambda \tau}-r \int_{0}^{+\infty} \kappa(s) e^{\lambda s} d s$.

Remark 2.6. The above result (2.11) on the impulsive delay differential inequality is an extension of continuous case in [37] and will play an important role in the following qualitative analysis of ISRDNNs with mixed time delays.

Lemma 2.7 (see [38]). Let $a, b \in \mathbb{R}^{n}$ and $X$ be an $n \times n$ positive definite matrix, then

$$
2 a^{T} b \leq a^{T} X a+b^{T} X^{-1} b .
$$

\section{Main Results}

This section deals with obtaining sufficient conditions that guarantee the existence and global exponential stability of periodic solution for the system (2.1)-(2.2).

Theorem 3.1. In addition to (A1)-(A5) and further assume that

$$
\text { (A6) } p>q+r \int_{0}^{+\infty} \kappa(s) d s \text {, }
$$


Abstract and Applied Analysis

(A7) there exist constants $M \geq 1, \lambda \in(0, \eta)$ and $\alpha \in[0, \lambda)$ such that

$$
\begin{gathered}
\prod_{k=1}^{n} \max \left\{1, \beta_{k}\right\} \leq M e^{\alpha t_{n}}, \quad n \in Z^{+}, \\
\lambda<p-q e^{\lambda \tau}-r \int_{0}^{+\infty} \kappa(s) e^{\lambda s} d s,
\end{gathered}
$$

where

$$
\begin{aligned}
& p= 2 \sum_{l=1}^{m} \frac{\min _{i \in N}\left(D_{i}\right)}{d_{l}^{2}}+2 \min _{i \in N}\left(\widehat{a}_{i}\right) \\
&-\left[\max _{i \in N}\left(\sum_{j=1}^{n}\left|\widehat{b}_{i j}\right| L_{j}^{f}\right)+\sum_{i=1}^{n} \max _{j \in N}\left(\left|\widehat{b}_{i j}\right| L_{j}^{f}\right)\right. \\
&\left.\quad+\max _{i \in N}\left(\sum_{j=1}^{n}\left|\widehat{\bar{b}}_{i j}\right| L_{j}^{\bar{f}}\right)+\max _{i \in N}\left(\sum_{j=1}^{n}\left|\widehat{\bar{b}}_{i j}\right| L_{j}^{\bar{f}}\right)+\max _{i \in N}\left\{\delta_{i}\right\}\right], \\
& q= {\left[\sum_{i=1}^{n} \max _{j \in N}\left(\left|\widehat{b}_{i j}\right| L_{j}^{f}\right)+\max _{i \in N}\left\{\gamma_{i}\right\}\right], } \\
& r= \sum_{i=1}^{n} \max _{j \in N}\left(\left|\widehat{\bar{b}}_{i j}\right| L_{j}^{\bar{f}}\right), \quad \beta_{k}=\max _{i \in N}\left\{\left(1-\theta_{i k}\right)^{2}\right\}, \\
& D_{i}=\min _{1 \leq l \leq m}\left\{D_{i l}\right\},
\end{aligned}
$$

then system (2.1)-(2.2) is globally exponentially periodic in the mean square sense.

Proof. For any $\psi=\left(\psi_{1}, \ldots, \psi_{n}\right)^{T}, \varphi=\left(\varphi_{1}, \ldots, \varphi_{n}\right)^{T} \in P \mathbb{C}_{F_{0}}^{b}$, let $\bar{u}(t)=\left(\bar{u}_{1}(t), \ldots, \bar{u}_{n}(t)\right)^{T}$ and $\underline{u}(t)=\left(\underline{u}_{1}(t), \ldots, \underline{u}_{n}(t)\right)^{T}$ be the solutions of system (2.1)-(2.2) starting from $\psi$ and $\varphi$, respectively.

Let $z_{i}(t)=\bar{u}_{i}(t)-\underline{u}_{i}(t)$, from $(2.1)$, we get

$$
\begin{aligned}
& d z_{i}(t)=\sum_{l=1}^{m} \frac{\partial}{\partial x_{l}}\left(D_{i l} \frac{\partial z_{i}(t)}{\partial x_{l}}\right) d t+-a_{i}(t) z_{i}(t)+\sum_{j=1}^{n} b_{i j}(t)\left(f_{j}\left(\bar{u}_{j}(t)\right)-f_{j}\left(\underline{u}_{j}(t)\right)\right) \\
&+\sum_{j=1}^{n} \tilde{b}_{i j}(t)\left(\tilde{f}_{j}\left(\bar{u}_{j}(t-\tau(t))\right)-\tilde{f}_{j}\left(\underline{u}_{j}(t-\tau(t))\right)\right) \\
&\left.+\sum_{j=1}^{n} \bar{b}_{i j}(t) \int_{-\infty}^{t} k_{i j}(t-s)\left(\bar{f}_{j}\left(\bar{u}_{j}(s)\right)-\bar{f}_{j}\left(\underline{u}_{j}(s)\right)\right) d s\right] d t \\
&+\sum_{j=1}^{n}\left[\sigma_{i j}\left(t, x, \bar{u}_{i}(t), \bar{u}_{i}(t-\tau(t))\right)-\sigma_{i j}\left(t, x, \underline{u}_{i}(t), \underline{u}_{i}(t-\tau(t))\right)\right] d w_{j}(t) .
\end{aligned}
$$

Construct the Lyapunov functional $V(t)=\int_{\Omega} \sum_{i=1}^{n} z_{i}^{2}(t) d x, i \in N$, 
for $t=t_{k}$, from (2.1) and (A4), we have

$$
\begin{aligned}
V\left(t_{k}\right) & =\int_{\Omega} \sum_{i=1}^{n} z_{i}^{2}\left(t_{k}\right) d x=\int_{\Omega} \sum_{i=1}^{n}\left[\bar{u}_{i}\left(t_{k}\right)-\underline{u}_{i}\left(t_{k}\right)\right]^{2} d x \\
& =\int_{\Omega} \sum_{i=1}^{n}\left[\bar{u}_{i}\left(t_{k}+\omega\right)-\underline{u}_{i}\left(t_{k}\right)\right]^{2} d x=\int_{\Omega} \sum_{i=1}^{n}\left(1-\theta_{i k}\right)^{2}\left[\bar{u}_{i}\left(t_{k+q}^{-}\right)-\underline{u}_{i}\left(t_{k}^{-}\right)\right]^{2} d x \\
& \leq \max _{i \in N}\left(1-\theta_{i k}\right)^{2} \int_{\Omega} \sum_{i=1}^{n}\left[\bar{u}_{i}\left(t_{k}^{-}+\omega\right)-\underline{u}_{i}\left(t_{k}^{-}\right)\right]^{2} d x=\max _{i \in N}\left(1-\theta_{i k}\right)^{2} V\left(t_{k}^{-}\right),
\end{aligned}
$$

when $t \in\left(t_{k-1}, t_{k}\right]$, the infinitesimal operator of $L V(t)$ along with system (3.3) is

$$
\begin{aligned}
& L V(t)=\int_{\Omega} 2 \sum_{i=1}^{n} z_{i}(t) \\
& \quad \times\left\{\sum_{l=1}^{m} \frac{\partial}{\partial x_{l}}\left(D_{i l} \frac{\partial z_{i}(t)}{\partial x_{l}}\right)-a_{i}(t) z_{i}(t)\right. \\
& \quad+\sum_{j=1}^{n} b_{i j}(t)\left[f_{j}\left(\bar{u}_{j}(t)\right)-f_{j}\left(\underline{u}_{j}(t)\right)\right]+\sum_{j=1}^{n} \tilde{b}_{i j}(t)\left(\tilde{f}_{j}\left(\bar{u}_{j}(t-\tau(t))\right)-\tilde{f}_{j}\left(\underline{u}_{j}(t-\tau(t))\right)\right) \\
& \left.\quad+\sum_{j=1}^{n} \bar{b}_{i j}(t) \int_{-\infty}^{t} k_{i j}(t-s)\left[\bar{f}_{j}\left(\bar{u}_{j}(s)\right)-\bar{f}_{j}\left(\underline{u}_{j}(s)\right)\right] d s\right\} d x \\
& +\int_{\Omega} \sum_{i=1}^{n}\left[\sigma_{i}\left(t, x, \bar{u}_{i}(t), \bar{u}_{i}(t-\tau(t))\right)-\sigma_{i}\left(t, x, \underline{u}_{i}(t), \underline{u}_{i}(t-\tau(t))\right)\right] \\
& \times\left[\sigma_{i}\left(t, x, \bar{u}_{i}(t), \bar{u}_{i}(t-\tau(t))\right)-\sigma_{i}\left(t, x, \underline{u}_{i}(t), \underline{u}_{i}(t-\tau(t))\right)\right]^{T} d x .
\end{aligned}
$$

Combining Cauchy inequality with (A2) yields

$$
\begin{aligned}
& \int_{\Omega} z_{i}(t) \int_{-\infty}^{t} K_{i j}(t-s)\left[\bar{f}_{j}\left(\bar{u}_{j}(t)\right)-\bar{f}_{j}\left(\underline{u}_{j}(t)\right)\right] d s d x \\
& \quad \leq \int_{\Omega}\left|z_{i}(t)\right| \int_{0}^{+\infty} K_{i j}(s) L_{j}^{\bar{f}}\left|z_{j}(t-s)\right| d s d x=\int_{0}^{+\infty} K_{i j}(s) L_{j}^{\bar{f}} \int_{\Omega}\left|z_{i}(t) \| z_{j}(t-s)\right| d x d s \\
& \quad \leq L_{j}^{\bar{f}}\left\|z_{i}(t)\right\|_{2} \int_{0}^{+\infty} K_{i j}(s) L_{j}^{\bar{f}}\left\|z_{j}(t-s)\right\|_{2} d s \leq \frac{1}{2} L_{j}^{\bar{f}}\left[\left\|z_{i}(t)\right\|_{2}^{2}+\left(\int_{0}^{+\infty} K_{i j}(s)\left\|z_{j}(t-s)\right\|_{2} d s\right)^{2}\right] \\
& \quad=\frac{1}{2} L_{j}^{\bar{f}}\left\|z_{i}(t)\right\|_{2}^{2}+\frac{1}{2} L_{j}^{\bar{f}}\left(\int_{0}^{+\infty}\left(K_{i j}(s)\right)^{1 / 2}\left(K_{i j}(s)\right)^{1 / 2}\left\|z_{j}(t-s)\right\|_{2} d s\right)^{2} \\
& \quad \leq \frac{1}{2} L_{j}^{\bar{f}}\left\|z_{i}(t)\right\|_{2}^{2}+\frac{1}{2} L_{j}^{\bar{f}}\left(\int_{0}^{+\infty} K_{i j}(s)\left\|z_{j}(t-s)\right\|_{2}^{2} d s\right) .
\end{aligned}
$$


According to Green's formula [37] and the Dirichlet boundary condition, we get

$$
\int_{\Omega} \sum_{l=1}^{m} z_{i}(t) \frac{\partial}{\partial x_{l}}\left(D_{i l} \frac{\partial z_{i}(t)}{\partial x_{l}}\right) d x=-\sum_{l=1}^{m} \int_{\Omega} D_{i l}\left(\frac{\partial z_{i}(t)}{\partial x_{l}}\right)^{2} d x
$$

Moreover, from Lemma 2.3, we have

$$
-\sum_{l=1}^{m} \int_{\Omega} D_{i l}\left(\frac{\partial z_{i}(t)}{\partial x_{l}}\right)^{2} d x \leq-\int_{\Omega} \sum_{l=1}^{m} \frac{D_{i l}}{d_{l}^{2}}\left(z_{i}(t)\right)^{2} d x \leq-\int_{\Omega} \sum_{l=1}^{m} \frac{\min _{i \in N}\left(D_{i}\right)}{d_{l}^{2}}\left(z_{i}(t)\right)^{2} d x
$$

From (A1)-(A3), (A5) and (3.5)-(3.8), we have

$$
\begin{aligned}
& L V(t) \leq-2 \sum_{l=1}^{m} \sum_{i=1}^{n}\left(\frac{D_{i l}}{d_{l}^{2}}\left\|z_{i}(t)\right\|_{2}^{2}\right) \\
& +2 \sum_{i=1}^{n}\left\{-\widehat{a}_{i}\left\|z_{i}(t)\right\|_{2}^{2}+\sum_{j=1}^{n}\left(\left|\widehat{b}_{i j}\right| L_{j}^{f}\left\|z_{i}(t)\right\|_{2}\left\|z_{j}(t)\right\|_{2}\right)+\frac{1}{2} \sum_{j=1}^{n}\left|\widehat{\bar{b}}_{i j}\right| L_{j}^{\bar{f}}\right. \\
& \times\left(\left\|z_{i}(t)\right\|_{2}^{2}+\left(\int_{0}^{+\infty} K_{i j}(s)\left\|z_{j}(t-s)\right\|_{2}^{2} d s\right)\right) \\
& \left.+\sum_{j=1}^{n}\left(\left|\widetilde{\tilde{b}}_{i j}\right| L_{j}^{\tilde{f}}\left\|z_{i}(t)\right\|_{2}\left\|z_{j}(t-\tau(t))\right\|_{2}\right)\right\}+\sum_{i=1}^{n}\left(\delta_{i}\left\|z_{i}(t)\right\|_{2}^{2}+\gamma_{i}\left\|z_{i}(t-\tau(t))\right\|_{2}^{2}\right) \\
& \leq-\sum_{l=1}^{m} \sum_{i=1}^{n}\left(\frac{2 D_{i l}}{d_{l}^{2}}\left\|z_{i}(t)\right\|_{2}^{2}\right) \\
& +\sum_{i=1}^{n}\left\{-2 \widehat{a}_{i}\left\|z_{i}(t)\right\|_{2}^{2}+\sum_{j=1}^{n}\left|\widehat{b}_{i j}\right| L_{j}^{f}\left(\left\|z_{i}(t)\right\|_{2}^{2}+\left\|z_{j}(t)\right\|_{2}^{2}\right)\right. \\
& +\sum_{j=1}^{n}\left|\hat{\bar{b}}_{i j}\right| L_{j}^{\bar{f}}\left(\left\|z_{i}(t)\right\|_{2}^{2}+\left(\int_{0}^{+\infty} K_{i j}(s)\left\|z_{j}(t-s)\right\|_{2}^{2} d s\right)\right) \\
& \left.+\sum_{j=1}^{n}\left[\left|\widehat{\tilde{b}}_{i j}\right| L_{j}^{\tilde{f}}\left(\left\|z_{i}(t)\right\|_{2}^{2}+\left\|z_{j}(t-\tau(t))\right\|_{2}^{2}\right)\right]\right\}+\sum_{i=1}^{n}\left(\delta_{i}\left\|z_{i}(t)\right\|_{2}^{2}+\gamma_{i}\left\|z_{i}(t-\tau(t))\right\|_{2}^{2}\right)
\end{aligned}
$$




$$
\begin{aligned}
& \leq\left\{-2 \sum_{l=1}^{m} \frac{\min _{i \in N}\left(D_{i}\right)}{d_{l}^{2}}-2 \min _{i \in N}\left(\widehat{a}_{i}\right)\right. \\
& +\left[\max _{i \in N}\left(\sum_{j=1}^{n}\left|\widehat{b}_{i j}\right| L_{j}^{f}\right)+\sum_{i=1}^{n} \max _{j \in N}\left(\left|\widehat{b}_{i j}\right| L_{j}^{f}\right)+\max _{i \in N}\left(\sum_{j=1}^{n}\left|\widehat{\widetilde{b}}_{i j}\right| L_{j}^{\tilde{f}}\right)\right. \\
& \left.\left.+\max _{i \in N}\left(\sum_{j=1}^{n}\left|\hat{\bar{b}}_{i j}\right| L_{j}^{\bar{f}}\right)+\max _{i \in N}\left\{\delta_{i}\right\}\right]\right\} \sum_{i=1}^{n}\left\|z_{i}(t)\right\|_{2}^{2}+\left[\sum_{i=1}^{n} \max _{j \in N}\left(\left|\hat{\tilde{b}}_{i j}\right| L_{j}^{\tilde{f}}\right)+\max _{i \in N}\left\{\gamma_{i}\right\}\right] \\
& \times \sum_{i=1}^{n}\left\|z_{i}(t-\tau(t))\right\|_{2}^{2}+\sum_{i=1}^{n} \max _{j \in N}\left(\left|\widehat{\bar{b}}_{i j}\right| L_{j}^{\bar{f}}\right) \int_{0}^{+\infty} \kappa(s) \sum_{i=1}^{n}\left\|z_{i}(t-s)\right\|_{2}^{2} d s .
\end{aligned}
$$

From (3.4), (3.9), (A6), (A7) and Lemma 2.5, we know

$$
E V(t) \leq M E V_{0} e^{-(\alpha-\beta) t}, \quad t \geq t_{0}
$$

which means that

$$
\int_{\Omega} \sum_{i=1}^{n} E\left[\bar{u}_{i}(t)-\underline{u}_{i}(t)\right]^{2} d x \leq M\|\varphi-\psi\|_{\tau}^{2} e^{-(\alpha-\beta) t}, \quad t \geq t_{0} .
$$

By the integral property of measurable functions, we can derive

$$
\int_{\Omega} \sum_{i=1}^{n}\left[\bar{u}_{i}(t+\omega)-\bar{u}_{i}(t)\right]^{2} d x \leq M\|\varphi-\psi\|^{2} e^{-(\alpha-\beta) t}, \quad t \geq t_{0} \text { a.e. }
$$

In the light of $\left(\sum_{i=1}^{n}\left|z_{i}\right|\right)^{2} \leq n \sum_{i=1}^{n}\left|z_{i}\right|^{2}$, for any $z_{i} \in \mathbb{R}^{+}$, we obtain

$$
\int_{\Omega} \sum_{i=1}^{n}\left|\bar{u}_{i}(t+\omega)-\bar{u}_{i}(t)\right| d x \leq \sqrt{n M}\|\varphi-\psi\| e^{-0.5(\alpha-\beta) t}, \quad t \geq t_{0} \text { a.e. }
$$

Noticing that

$$
\bar{u}_{i}(t+k \omega)=\bar{u}_{i}(t)+\sum_{r=1}^{k}\left[\bar{u}_{i}(t+r \omega)-\bar{u}_{i}(t+(r-1) \omega)\right], \quad i \in N .
$$


For any given $t \geq t_{0}$, by (3.12), we can see that

$$
\begin{aligned}
& \int_{\Omega} \sum_{r=1}^{\infty}\left[\bar{u}_{i}(t+r \omega)-\bar{u}_{i}(t+(r-1) \omega)\right] d x \\
& \quad=\int_{\Omega} \lim _{k \rightarrow \infty} \sum_{r=1}^{k}\left[\left(\overline{\mathcal{u}}_{i}(t+r \omega)-\bar{u}_{i}(t+(r-1) \omega)\right)\right] d x \leq \sqrt{n M}\|\varphi-\psi\| \lim _{k \rightarrow \infty} \sum_{r=1}^{k} e^{-0.5(\alpha-\beta)(t+(r-1) \omega)} \\
& \quad \leq \sqrt{n M}\|\varphi-\psi\| e^{-0.5(\alpha-\beta) t} \lim _{k \rightarrow \infty} \sum_{r=1}^{k} e^{-0.5(\alpha-\beta)(r-1) \omega}
\end{aligned}
$$

therefore, $\lim _{k \rightarrow \infty} \bar{u}_{i}(t+k \omega)$ exists a.e.

Let $\widehat{u}(t)=\left(\widehat{u}_{1}(t), \ldots, \widehat{u}_{n}(t)\right)^{T}$ be the solution of system (2.1)-(2.2) starting from $\phi$, by $\widehat{u}_{i}(t)=\lim _{k \rightarrow \infty} \bar{u}_{i}(t+k \omega)$, then $\widehat{u}(t)$ is well defined and is a periodic function with period $\omega$. Supposing that $\widehat{v}(t)=\left(\widehat{v}_{1}(t), \ldots, \widehat{v}_{n}(t)\right)^{T}$ is another $\omega$-periodic solution of system (2.1)-(2.2) starting from $\phi^{*}$, by similar method used before, it is easy to prove

$$
\begin{aligned}
\int_{\Omega} \sum_{i=1}^{n}\left[\widehat{u}_{i}(t)-\widehat{v}_{i}(t)\right]^{2} d x & =\int_{\Omega} \sum_{i=1}^{n}\left[\widehat{u}_{i}(t+k \omega)-\widehat{v}_{i}(t+k \omega)\right]^{2} d x \\
& \leq M\left\|\phi-\phi^{*}\right\|^{2} e^{-(\alpha-\beta)(t+k \omega)}, \quad t \geq t_{0}, \text { a.e. }
\end{aligned}
$$

Therefore, we can conclude that the system (2.1)-(2.2) is globally exponentially periodic in the mean square sense. This completes the proof of Theorem 3.1.

Next, omitting condition (A4) and using LMI technique, another sufficient condition ensuring the global exponential stability of periodic solution for the system (2.1)-(2.2) in the mean square sense is derived.

Theorem 3.2. Suppose that (A1)-(A3) and (A5) hold. If there exists a positive definite diagonal matrix $P$, positive definite matrices $\Xi_{1}, \Xi_{2}$, nonnegative constants $p, q, r$, and $\beta_{k},\left(k \in Z^{+}\right)$, such that

(i) $p>q+r \int_{0}^{+\infty} \kappa(s) d s$,

(ii) there exist constants $M \geq 1, \lambda \in(0, \eta)$ and $\alpha \in[0, \lambda)$ such that

$$
\prod_{k=1}^{n} \max \left\{1, \beta_{k}\right\} \leq M e^{\alpha t_{n}}, \quad n \in Z^{+},
$$

$$
\text { and } \lambda<p-q e^{\lambda \tau}-r \int_{0}^{+\infty} \kappa(s) e^{\lambda s} d s
$$


(iii)

$$
\begin{aligned}
& -P D^{*}-D^{* T} P-P A-A^{T} P+P B L^{f}+L^{f} B^{T} P+R_{1}+P \tilde{B} L^{\tilde{f}} \Xi_{1} L^{\tilde{f}} \widetilde{B}^{T} P \\
& +\int_{0}^{+\infty} \kappa(s) d s P \bar{B} L^{\bar{f}} \Xi_{2} L^{\bar{f}} \bar{B}^{T} P+p P<0, \quad \Xi_{1}^{-1}+R_{2}-q P<0, \Xi_{2}^{-1}-r P<0, C_{k}^{T} P C_{k}-\beta_{k} P<0,
\end{aligned}
$$

then the system (2.1)-(2.2) is global exponential periodic in the mean square sense. Where

$$
\begin{gathered}
A=\operatorname{diag}\left(a_{1}, \ldots, a_{n}\right), \quad B=\left(b_{i j}\right)_{n \times n^{\prime}} \quad \tilde{B}=\left(\tilde{b}_{i j}\right)_{n \times n^{\prime}} \quad \bar{B}=\left(\bar{b}_{i j}\right)_{n \times n^{\prime}} \\
\beta_{k}=\max _{i \in N}\left\{\left(1-\theta_{i k}\right)^{2}\right\}, \quad R_{1}=\operatorname{diag}\left(\delta_{1}, \ldots, \delta_{n}\right), \quad R_{2}=\operatorname{diag}\left(\gamma_{1}, \ldots, \gamma_{n}\right), \\
D^{*}=\operatorname{diag}\left(\sum_{l=1}^{m} \frac{D_{1 l}}{d_{l}^{2}}, \ldots, \sum_{l=1}^{m} \frac{D_{n l}}{d_{l}^{2}}\right), \quad C_{k}=\operatorname{diag}\left(1-\theta_{1 k}, \ldots, 1-\theta_{n k}\right) .
\end{gathered}
$$

Proof. Define the following Lyapunov functional:

$$
V(t)=\int_{\Omega} z^{T}(t) P z(t) d x
$$

when $t=t_{k}$, we have

$$
\begin{aligned}
V\left(t_{k}\right)-\beta_{k} V\left(t_{k}^{-}\right) & =\int_{\Omega} z^{T}\left(t_{k}^{-}\right) C_{k}^{T} P C_{k} z\left(t_{k}^{-}\right)-z^{T}\left(t_{k}^{-}\right) \beta_{k} P z\left(t_{k}^{-}\right) d x \\
& =\int_{\Omega} z^{T}\left(t_{k}^{-}\right)\left(C_{k}^{T} P C_{k}-\beta_{k} P\right) z\left(t_{k}^{-}\right) d x<0 .
\end{aligned}
$$

For $t \geq t_{0}, t \neq t_{k}$, the infinitesimal operator of $L V(t)$ along with (3.16) is

$$
\begin{array}{r}
L V(t)=\int_{\Omega}\left(\frac{\partial}{\partial t} z^{T} P z+z^{T} P \frac{\partial z}{\partial t}\right) d x+\int_{\Omega} \operatorname{trace}\left(\sigma^{T} P \sigma\right) d x \\
\leq 2 \int_{\Omega} z^{T} P\left(\sum_{l=1}^{m} \frac{\partial}{\partial x_{l}}\left(D_{i l} \frac{\partial z}{\partial x_{l}}\right)-A z(t)+B g(z(t))+\tilde{B} \tilde{g}(z(t-\tau(t)))\right. \\
\left.\quad+\bar{B} \int_{-\infty}^{t} \kappa(t-s) L^{\bar{f}} z(s) d s\right) d x+\int_{\Omega} \operatorname{trace}\left(\tilde{\sigma}^{T} P \tilde{\sigma}\right) d x
\end{array}
$$


where

$$
\begin{gathered}
g(z(t))=\left(g_{1}\left(z_{1}(t)\right), \ldots, g_{n}\left(z_{n}(t)\right)\right)^{T}, \quad \tilde{\sigma}=\left(\sigma_{i j}\left(t, x, \xi_{i}^{\prime}, \varsigma_{i}^{\prime}\right)-\sigma_{i j}\left(t, x, \xi_{i}, \varsigma_{i}\right)\right)_{n \times n} \\
\tilde{g}(z(t))=\left(\tilde{g}_{1}\left(z_{1}(t-\tau(t))\right), \ldots, \tilde{g}_{n}\left(z_{n}(t-\tau(t))\right)\right)^{T}, \\
\bar{g}(z(s))=\left(\bar{g}_{1}\left(z_{1}(s)\right), \ldots, \bar{g}_{n}\left(z_{n}(s)\right)\right)^{T}, \quad g_{j}\left(z_{j}(t)\right)=f_{j}\left(\bar{u}_{j}(t)\right)-f_{j}\left(\underline{u}_{j}(t)\right), \\
\tilde{g}_{j}\left(z_{j}(t-\tau(t))\right)=\tilde{f}_{j}\left(\bar{u}_{j}(t-\tau(t))\right)-\tilde{f}_{j}\left(\underline{u}_{j}(t-\tau(t))\right), \\
\bar{g}_{j}\left(z_{j}(s)\right)=\bar{f}_{j}\left(\bar{u}_{j}(s)\right)-\bar{f}_{j}\left(\underline{u}_{j}(s)\right), \quad j=1,2, \ldots, n .
\end{gathered}
$$

By employing (3.8), (A5) and Lemma 2.7, we have

$$
\begin{aligned}
& L V \leq 2 \int_{\Omega}\left(-z^{T}(t) P D^{*} z(t)-z^{T}(t) P A z(t)+z^{T}(t) P B L^{f} z(t)\right. \\
& \left.+z^{T}(t) P \widetilde{B} L^{\tilde{f}} z(t-\tau(t))+z^{T}(t) P \bar{B} \int_{0}^{+\infty} \kappa(s) L^{\bar{f}} z(t-s) d s\right) d x \\
& +\int_{\Omega}\left(z^{T}(t) R_{1} z(t)+z^{T}(t-\tau(t)) R_{2} z(t-\tau(t))\right) d x \\
& \leq \int_{\Omega}\left[z^{T}(t)\left(-P D^{*}-D^{* T} P-P A-A^{T} P+P B L^{f}+L^{f} B^{T} P+R_{1}\right) z(t)\right. \\
& +z^{T}(t) P \widetilde{B} L^{\tilde{f}} \Xi_{1} L^{\tilde{f}} \widetilde{B}^{T} P z(t)+z^{T}(t-\tau(t)) \Xi_{1}^{-1} z(t-\tau(t)) \\
& \left.+\int_{0}^{+\infty} \kappa(s) z^{T}(t) P \bar{B} L^{\bar{f}} \Xi_{2} L^{\bar{f}} \bar{B}^{T} P z(t) d s\right] d x \\
& +\int_{\Omega}\left(z^{T}(t-\tau(t)) R_{2} z(t-\tau(t))+\int_{0}^{+\infty} \kappa(s) z^{T}(t-s) \Xi_{2}^{-1} z(t-s) d s\right) d x \\
& \leq \int_{\Omega}\left[z ^ { T } ( t ) \left(-P D^{*}-D^{* T} P-P A-A^{T} P+P B L^{f}\right.\right. \\
& \left.+L^{f} B^{T} P+R_{1}+P \widetilde{B} L^{\tilde{f}} \Xi_{1} L^{\tilde{f}} \widetilde{B}^{T} P+\int_{0}^{+\infty} \kappa(s) d s P \bar{B} L^{\bar{f}} \Xi_{2} L^{\bar{f}} \bar{B}^{T} P\right) z(t) \\
& \left.+z^{T}(t-\tau(t))\left(\Xi_{1}^{-1}+R_{2}\right) z(t-\tau(t))+\int_{0}^{+\infty} \kappa(s) z^{T}(t-s) \Xi_{2}^{-1} z(t-s) d s\right] d x \\
& \leq \int_{\Omega}\left[z ^ { T } ( t ) \left(-P D^{*}-D^{* T} P-P A-A^{T} P+P B L^{f}+L^{f} B^{T} P\right.\right. \\
& \left.+R_{1}+P \widetilde{B} L^{\tilde{f}} \Xi_{1} L^{\tilde{f}} \widetilde{B}^{T} P+\int_{0}^{+\infty} \kappa(s) d s P \bar{B} L^{\bar{f}} \Xi_{2} L^{\bar{f}} \bar{B}^{T} P+p P\right) z(t)
\end{aligned}
$$




$$
\begin{aligned}
& \left.+z^{T}(t-\tau(t))\left(\Xi_{1}^{-1}+R_{2}-q P\right) z(t-\tau(t))+\int_{0}^{+\infty} \kappa(s) z^{T}(t-s)\left(\Xi_{2}^{-1}-r P\right) z(t-s) d s\right] d x \\
& -p V(t)+q \sup _{-\infty \leq s \leq t} V(s)+r \int_{0}^{+\infty} \kappa(s) V(t-s) d s .
\end{aligned}
$$

It follows from the condition (iii) and (3.24) that we have

$$
L V(t) \leq-p V(t)+q \sup _{-\infty \leq s \leq t} V(t)+r \int_{0}^{+\infty} \kappa(s) V(t-s) d s
$$

By Lemma 2.5, we obtain

$$
\lambda_{\min }(P) E\|z(t)\|_{2}^{2} \leq E V(t) \leq \lambda_{\max }(P)\|\psi-\varphi\|_{\tau}^{2} e^{-(\lambda-\alpha) t} .
$$

We know that

$$
E\|z(t)\|_{2} \leq \sqrt{\frac{\lambda_{\max }(P)}{\lambda_{\min }(P)}}\|\psi-\varphi\|_{\tau} e^{-((\lambda-\alpha) t) / 2},
$$

that is,

$$
E\left\|\bar{u}_{i}(t)-\underline{u}_{i}(t)\right\|_{2} \leq \sqrt{\frac{\lambda_{\max }(P)}{\lambda_{\min }(P)}}\|\psi-\varphi\|_{\tau} e^{-((\lambda-\alpha) t) / 2}, \quad \forall t \geq t_{0} \geq 0,
$$

where

$$
M=\sqrt{\frac{\lambda_{\max }(P)}{\lambda_{\min }(P)}} \geq 1 .
$$

Similar to the proof of Theorem 3.1, we know that the system (2.1)-(2.2) is globally exponentially periodic in the mean square sense. This completes the proof.

Remark 3.3. In [23], the authors have considered the stability problems of RDNNs, however, they have not considered impulsive stochastic effect and reaction-diffusion terms. To the best of our knowledge, no LMI-based stability results have been reported for ISRDNNs with mixed time delays in the literature.

Since an equilibrium point can be viewed as a special periodic solution of RDNNs with arbitrary period, we can consider ISRDNNs in system (2.1) with parameters $a_{i}(t)=a_{i}$, $b_{i j}(t)=b_{i j}, \widetilde{b}_{i j}(t)=\tilde{b}_{i j}, \bar{b}_{i j}(t)=\bar{b}_{i j}, J_{i}(t)=J_{i}, \tau(t)=\tau, \sigma_{i j}\left(t, x, u^{*}, u^{*}\right)=0$, where $a_{i}, b_{i j}, \tilde{b}_{i j}$, $\bar{b}_{i j}, J_{i}$ are constants. Then, according to the results obtained so far, if the sufficient conditions in Theorems 3.1 or 3.2 are satisfied, a unique periodic solution becomes a periodic solution with arbitrary positive constants as its period. So, the periodic solution reduces to a constant 
solution, that is, an equilibrium point. Moreover, all other solutions globally exponentially converge to this equilibrium point in the mean square sense as $t \rightarrow+\infty$. To this end, by applying Theorems 3.1 or 3.2, we can easily get the following results.

Corollary 3.4. Suppose that (A1)-(A5) hold for ISRDNNs in (2.1)-(2.2) with parameters $a_{i}(t)=$ $a_{i}, b_{i j}(t)=b_{i j}, \tilde{b}_{i j}(t)=\tilde{b}_{i j}, \bar{b}_{i j}(t)=\bar{b}_{i j}, J_{i}(t)=J_{i}, \tau(t)=\tau, \sigma_{i j}\left(t, x, u^{*}, u^{*}\right)=0$, where $a_{i}, b_{i j}$, $\tilde{b}_{i j}, \bar{b}_{i j}, J_{i}$ are constants, if $\theta_{i k} \in[0,2], i \in N, k \in Z^{+}$, then there exists a unique equilibrium point of system (2.1)-(2.2), which is globally exponentially stable in the mean square sense.

Corollary 3.5. Suppose that (A2)-(A3), (A5) for system (2.1)-(2.2) with $a_{i}, b_{i j}, \tilde{b}_{i j}, \bar{b}_{i j}, J_{i}$ being constants and $\theta_{i k} \in[0,2], i \in N, k \in Z^{+}$hold. If there exist a positive definite diagonal matrix $P$, positive definite matrices $\Xi_{1}, \Xi_{2}$, nonnegative constants $p, q, r$, and $\beta_{k},\left(k \in Z^{+}\right)$, such that (i) $p>q+r \int_{0}^{+\infty} \kappa(s) d s$,

(ii) there exist constants $M \geq 1, \lambda \in(0, \eta)$ and $\alpha \in[0, \lambda)$ such that

$$
\prod_{k=1}^{n} \max \left\{1, \beta_{k}\right\} \leq M e^{\alpha t_{n}}, \quad n \in Z^{+},
$$

and $\lambda<p-q e^{\lambda \tau}-r \int_{0}^{+\infty} \kappa(s) e^{\lambda s} d s$,

(iii)

$$
\begin{array}{r}
-P D^{*}-D^{* T} P-P A-A^{T} P+P B L^{f}+L^{f} B^{T} P+R_{1}+P \tilde{B} L^{\tilde{f}} \Xi_{1} L^{\tilde{f}} \widetilde{B}^{T} P \\
+\int_{0}^{+\infty} \kappa(s) d s P \bar{B} L^{\bar{f}} \Xi_{2} L^{\bar{f}} \bar{B}^{T} P+p P<0, \quad \Xi_{1}^{-1}+R_{2}-q P<0, \quad \Xi_{2}^{-1}-r P<0, \\
C_{k}^{T} P C_{k}-\beta_{k} P<0,
\end{array}
$$

then the system (2.1)-(2.2) has a unique equilibrium point, which is globally exponentially stable in the mean square sense.

\section{Illustrative Examples}

Example 4.1. Consider the system (2.1) with two neurons on $\Omega=\left\{\left(x_{1}, x_{2}\right)^{T} \mid 0<x_{l}<1, l=\right.$ $1,2\} \subset \mathbb{R}^{2}$, the boundary conditions and initial conditions are given by

$$
\begin{gathered}
u_{i}(t, x)=0, \quad(t, x) \in[0,+\infty) \times \partial \Omega, \\
u_{i}(s, x)=2 \sin \pi x_{1} x_{2}^{2}, \quad i=1,2, \quad(s, x) \in(-\infty, 0] \times \Omega,
\end{gathered}
$$

where $t_{k}=k, k \in Z^{+}, \kappa(s)=k_{i j}(s)=s e^{-s}, f_{j}(\eta)=\tilde{f}_{j}(\eta)=\bar{f}_{j}(\eta)=(1 / 30)(|\eta+1|+|\eta-1|), n=$ $m=2, L_{j}^{f}=L_{j}^{\tilde{f}}=L_{j}^{\bar{f}}=1, d_{l}=\varepsilon_{i}=1, j, l=1,2 . D_{11}=D_{12}=0.5, D_{21}=0.3, D_{22}=0.7, \tau(t)=$ 
$0.02-0.01 \sin 2 \pi t, \tau=\ln 2, a_{1}(t)=10.9-4 \cos 2 \pi t, a_{2}(t)=11-\sin 2 \pi t, \theta_{i k}=-1+k, k \in$ $Z^{+}, \delta_{i}=\gamma_{i}=1$.

$$
\begin{array}{cl}
\sigma_{i j}\left(t, x, u_{i}(t, x), u_{i}(t-\tau(t), x)\right)=\frac{\sqrt{2}}{2}\left(\tanh \left(u_{i}(t, x)\right)+\tanh \left(u_{i}(t-\tau(t), x)\right)\right), \\
b_{11}(t)=0.3+0.1 \sin 2 \pi t, \quad b_{12}(t)=0.4+0.1 \sin 2 \pi t, \quad b_{21}(t)=0.2+0.1 \cos 2 \pi t, \\
b_{22}(t)=0.3-0.1 \cos 2 \pi t, \quad \tilde{b}_{11}(t)=0.2+0.1 \sin 2 \pi t, \quad \tilde{b}_{12}(t)=0.3-0.2 \cos 2 \pi t, \\
\tilde{b}_{21}(t)=0.5+0.1 \cos 2 \pi t, \quad \tilde{b}_{22}(t)=0.4-0.1 \sin 2 \pi t, \quad \bar{b}_{11}(t)=0.1-0.2 \sin 2 \pi t, \\
\bar{b}_{12}(t)=0.25-0.1 \sin 2 \pi t, \quad \bar{b}_{21}(t)=0.2-0.1 \cos 2 \pi t, \quad \bar{b}_{22}(t)=0.1-0.1 \cos 2 \pi t, \\
J_{1}(t)=1+\sin 2 \pi t, \quad J_{2}(t)=2+\cos 2 \pi t .
\end{array}
$$

Direct computation shows that $p=5.65, q+r \int_{0}^{+\infty} \kappa(s) d s=4.45$. Let $\lambda=0.2, \alpha=0, M=1$, and $\tau=\ln 2$ satisfying $\lambda<p-q e^{\lambda \tau}-r \int_{0}^{+\infty} \kappa(s) e^{\lambda s} d s$. The simulation results are shown in Figures 1-6. When $x_{2}=0.1$, the states surfaces of $u\left(t, x_{1}, 0.1\right)$ are shown in Figures 1 and 2 , while $x_{1}=0.1$, the states surfaces of $u\left(t, 0.1, x_{2}\right)$ are shown in Figures 3 and 4 , they are illustrated that the system states in (2.1) and (2.2) converge to periodic solutions. In order to see it clearly, we also draw the curves of the states when $x_{1}=0.1, x_{2}=0.1$ in Figures 5 and 6 . Hence, it follows from both Theorem 3.1 and the simulation study that system (2.1)-(2.2) is globally exponentially periodic stable in the mean square sense.

Example 4.2. Consider an ISRDNNs in (2.1) with parameters on $\Omega=\left\{\left(x_{1}, x_{2}\right)^{T} \mid 0<x_{l}<\right.$ $1 / 2, l=1,2\}$,

$$
\begin{gathered}
t_{k}=0.5 k, \quad \mathcal{K}(s)=k_{i j}(s)=s e^{-s}, \quad D_{i l}=\frac{1}{8} \quad(i, j, l=1,2), \quad J_{1}(t)=\sin t, \quad J_{2}(t)=\cos t, \\
A=\left[\begin{array}{ll}
2 & 0 \\
0 & 2
\end{array}\right], \quad B=\left[\begin{array}{cc}
0.5 & -0.5 \\
0.5 & 0.5
\end{array}\right], \quad \bar{B}=\widetilde{B}=\left[\begin{array}{ll}
0.25 & 0.25 \\
0.25 & 0.25
\end{array}\right], \quad R_{1}=R_{2}=D^{*}=\left[\begin{array}{ll}
1 & 0 \\
0 & 1
\end{array}\right], \\
f_{j}(\eta)=\tilde{f}_{j}(\eta)=\bar{f}_{j}(\eta)=\sin \frac{\eta}{2}+\frac{\eta}{2}, \quad j=1,2, \quad \tau(t)=0.1-0.1 \sin t .
\end{gathered}
$$

Clearly, $f_{j}(\eta), \tilde{f}_{j}(\eta), \bar{f}_{j}(\eta),(j=1,2)$ satisfy the (A2) with $L^{f}=L^{\tilde{f}}=L^{\bar{f}}=I_{2}$, and $\tau(t), J_{1}(t), J_{2}(t)$ are continuously periodic functions with a common positive period $2 \pi$.

Taking $p=1, q=0.2, r=0.1, \lambda=0.1, \alpha=0, \beta_{k}=1, P=2 I_{2}, C_{k}=0.5 I_{2}, \Xi_{1}=\Xi_{2}=$ $I_{2}$. By simple calculation, we can easily check (i), (ii), (iii), and (iv) in Theorem 3.2.

To this end, the conditions of Theorem 3.2 are satisfied, therefore, there exists exactly one $2 \pi$-periodic solution, and all other solutions converge exponentially to it in the mean square sense as $t \rightarrow+\infty$.

Remark 4.3. In Examples 4.1 and 4.2, many factors such as noise perturbations, mixed time delays, and impulsive effects are considered. Therefore, the results reported in [13, 14, 18-20] do not hold in our examples. 


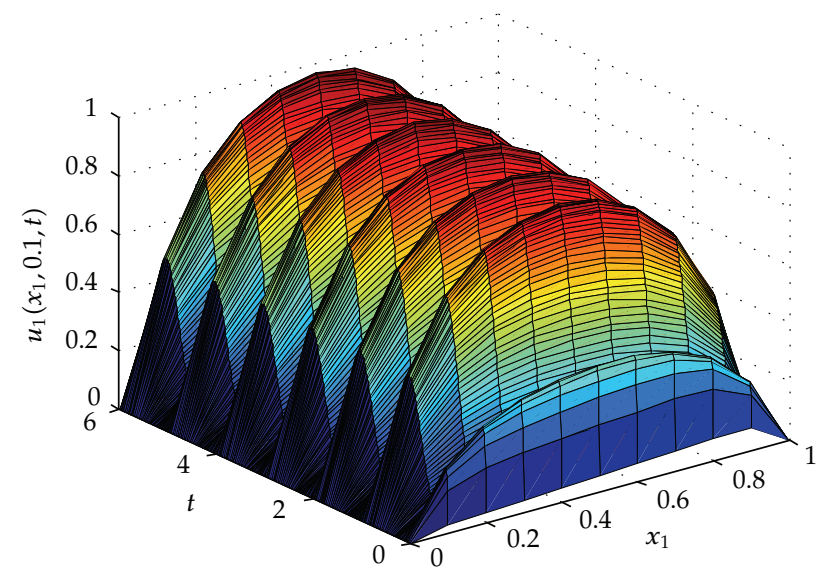

Figure 1: The surface of $u_{1}\left(t, x_{1}, 0.1\right)$ when $x_{2}=0.1$.

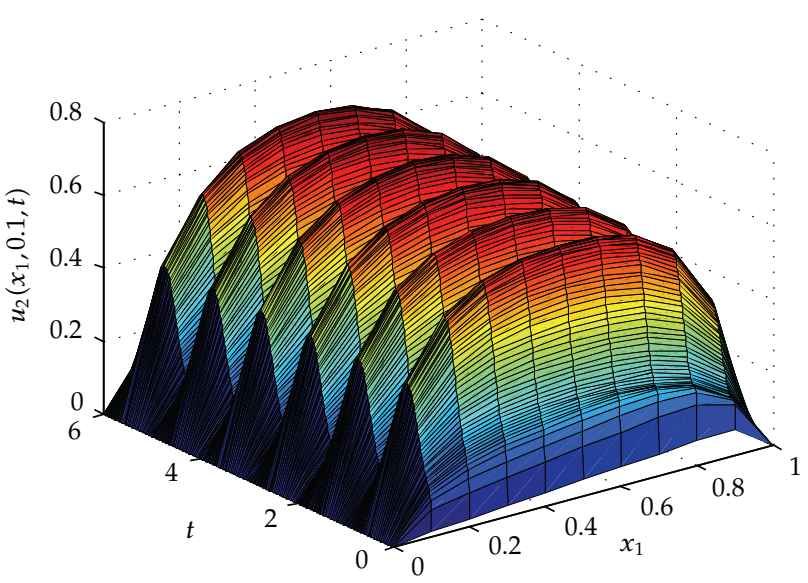

Figure 2: The surface of $u_{2}\left(t, x_{1}, 0.1\right)$ when $x_{2}=0.1$.

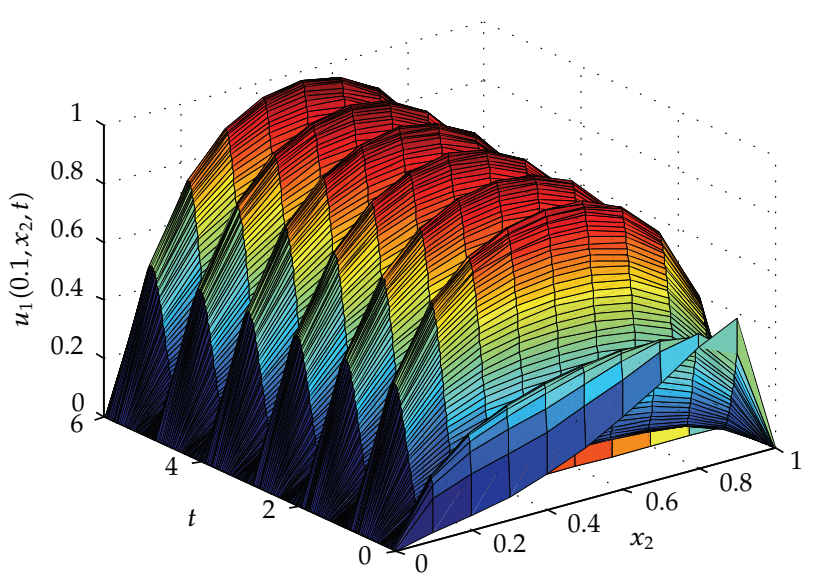

Figure 3: The surface of $u_{1}\left(t, 0.1, x_{2}\right)$ when $x_{1}=0.1$. 


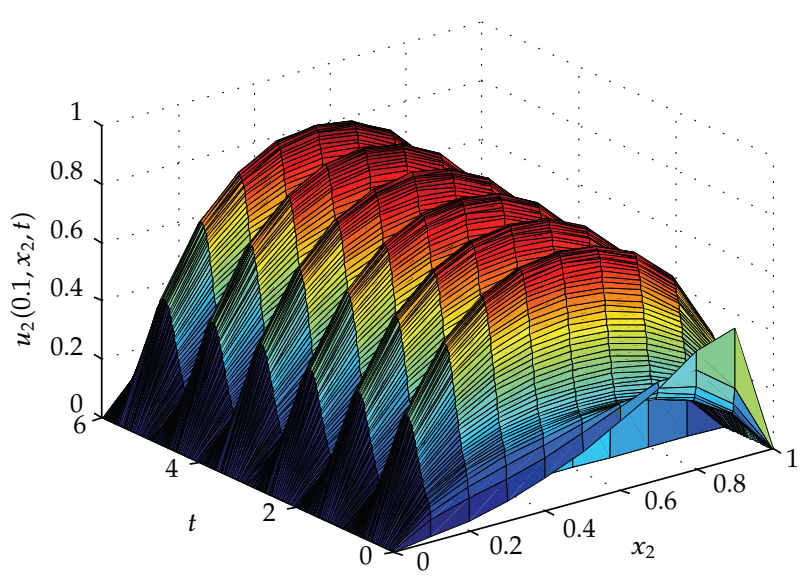

Figure 4: The surface of $u_{2}\left(t, 0.1, x_{2}\right)$ when $x_{1}=0.1$.

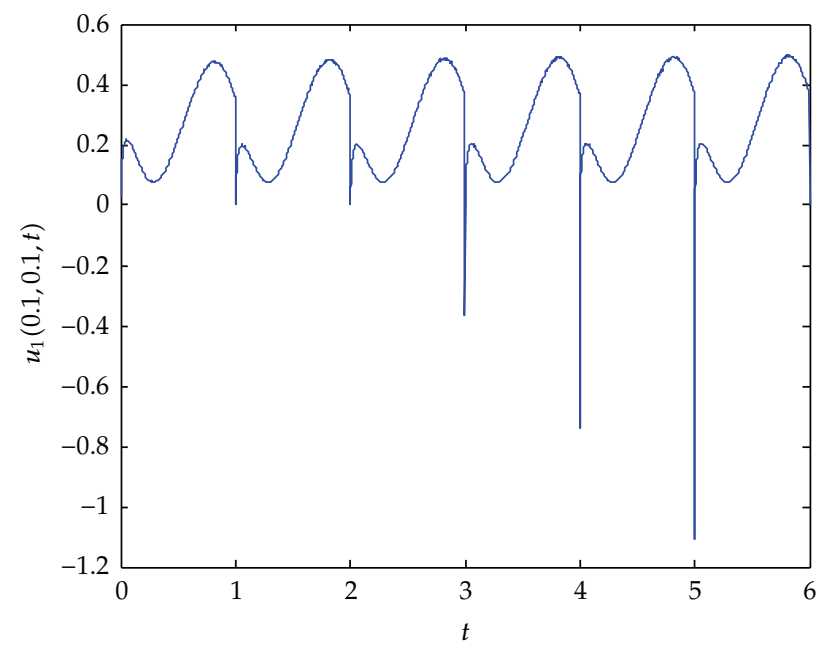

Figure 5: The curve of $u_{1}(t, 0.1,0.1)$ when $x_{1}=0.1, x_{2}=0.1$.

\section{Conclusions}

In this paper, the dynamical behaviors for ISRDNNs with mixed time delays have been studied. By using an L-operator differential inequality with impulses and mixed time delays, as well as linear matrix inequality technique, some novel sufficient conditions are derived to guarantee the existence, uniqueness, global exponential stability of the periodic solutions, and the global exponential stability of the equilibrium point in the mean square sense. To the best of our knowledge, the results presented here have been not appeared in the related literature. The obtained sufficient conditions depend on the reaction-diffusion terms. The obtained results generalize and comprise those results with/without reaction-diffusion term, impulsive operators, or noise disturbances in the previous literature. Finally, two numerical examples are also provided in the end of the paper to show the effectiveness of our results. 


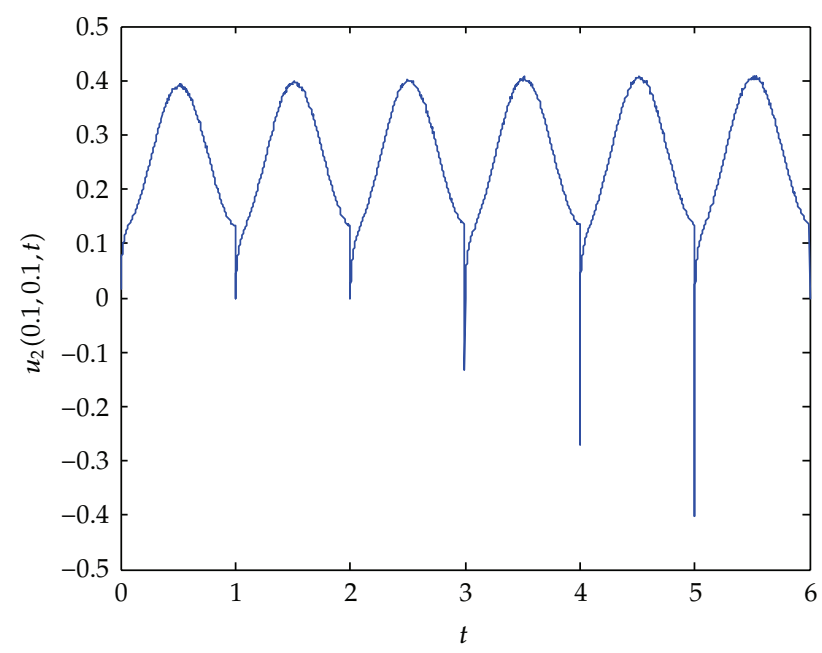

Figure 6: The curve of $u_{2}(t, 0.1,0.1)$ when $x_{1}=0.1, x_{2}=0.1$.

\section{Acknowledgments}

This work is partially supported by the National Natural Science Foundation of China under Grant no. 60974139 and partially supported by the Fundamental Research Funds for the Central Universities under Grant no. 72103676, the Special research projects in Shaanxi Province Department of Education under Grant nos. 2010JK896, 01JK060 and Research Projects of Xianyang Normal University under Grant no. 07XSYK284.

\section{References}

[1] Y. Zheng and T. Chen, "Global exponential stability of delayed periodic dynamical systems," Physics Letters A, vol. 322, no. 5-6, pp. 344-355, 2004.

[2] J. Cao and J. Wang, "Global exponential stability and periodicity of recurrent neural networks with time delays," IEEE Transactions on Circuits and Systems I, vol. 52, no. 5, pp. 920-931, 2005.

[3] P. Balasubramaniam and R. Rakkiyappan, "Delay-dependent robust stability analysis for Markovian jumping stochastic Cohen-Grossberg neural networks with discrete interval and distributed timevarying delays," Nonlinear Analysis. Hybrid Systems, vol. 3, no. 3, pp. 207-214, 2009.

[4] X. Li, "Existence and global exponential stability of periodic solution for delayed neural networks with impulsive and stochastic effects," Neurocomputing, vol. 73, no. 4-6, pp. 749-758, 2010.

[5] S. M. Lee, O. M. Kwon, and J. H. Park, "A novel delay-dependent criterion for delayed neural networks of neutral type," Physics Letters A, vol. 374, no. 17-18, pp. 1843-1848, 2010.

[6] C. Huang and J. Cao, "Convergence dynamics of stochastic Cohen-Grossberg neural networks with unbounded distributed delays," IEEE Transactions on Neural Networks, vol. 22, no. 4, pp. 561-572, 2011.

[7] Q. Zhu and J. Cao, "Stability of Markovian jump neural networks with impulse control and time varying delays," Nonlinear Analysis: Real World Applications, vol. 13, pp. 2259-2270, 2012.

[8] M. Forti and A. Tesi, "New conditions for global stability of neural networks with application to linear and quadratic programming problems," IEEE Transactions on Circuits and Systems I, vol. 42, no. 7, pp. 354-366, 1995.

[9] X. G. Liu, R. R. Martin, M. Wu, and M. L. Tang, "Global exponential stability of bidirectional associative memory neural networks with time delays," IEEE Transactions on Neural Networks, vol. 19, no. 3, pp. 397-407, 2008.

[10] Q. Zhu, C. Huang, and X. Yang, "Exponential stability for stochastic jumping BAM neural networks with time-varying and distributed delays," Nonlinear Analysis: Hybrid Systems, vol. 5, no. 1, pp. 52-77, 2011. 
[11] O. Faydasicok and S. Arik, "Equilibrium and stability analysis of delayed neural networks under parameter uncertainties," Applied Mathematics and Computation, vol. 218, no. 12, pp. 6716-6726, 2012.

[12] O. Faydasicok and S. Arik, "Further analysis of global robust stability of neural networks with multiple time delays," Journal of the Franklin Institute, vol. 349, no. 3, pp. 813-825, 2012.

[13] Q. Song, J. Cao, and Z. Zhao, "Periodic solutions and its exponential stability of reaction-diffusion recurrent neural networks with continuously distributed delays," Nonlinear Analysis: Real World Applications, vol. 7, no. 1, pp. 65-80, 2006.

[14] H. Zhao and G. Wang, "Existence of periodic oscillatory solution of reaction-diffusion neural networks with delays," Physics Letters A, vol. 343, no. 5, pp. 372-383, 2005.

[15] X. Zhang, S. Wu, and K. Li, "Delay-dependent exponential stability for impulsive Cohen-Grossberg neural networks with time-varying delays and reaction-diffusion terms," Communications in Nonlinear Science and Numerical Simulation, vol. 16, no. 3, pp. 1524-1532, 2011.

[16] P. Balasubramaniam and C. Vidhya, "Exponential stability of stochastic reaction-diffusion uncertain fuzzy neural networks with mixed delays and Markovian jumping parameters," Expert Systems With Applications, vol. 39, pp. 3109-3115, 2012.

[17] J. Pan, X. Liu, and S. Zhong, "Stability criteria for impulsive reaction-diffusion Cohen-Grossberg neural networks with time-varying delays," Mathematical and Computer Modelling, vol. 51, no. 9-10, pp. 1037-1050, 2010.

[18] J. Pan and S. Zhong, "Dynamical behaviors of impulsive reaction-diffusion Cohen-Grossberg neural network with delays," Neurocomputing, vol. 73, no. 7-9, pp. 1344-1351, 2010.

[19] J. G. Lu and L. J. Lu, "Global exponential stability and periodicity of reaction-diffusion recurrent neural networks with distributed delays and Dirichlet boundary conditions," Chaos, Solitons and Fractals, vol. 39, no. 4, pp. 1538-1549, 2009.

[20] J. G. Lu, "Global exponential stability and periodicity of reaction-diffusion delayed recurrent neural networks with Dirichlet boundary conditions," Chaos, Solitons and Fractals, vol. 35, no. 1, pp. 116-125, 2008.

[21] J. G. Lu, “Robust global exponential stability for interval reaction-diffusion Hopfield neural networks with distributed delays," IEEE Transactions on Circuits and Systems II, vol. 54, no. 12, pp. 1115-1119, 2007.

[22] Y. Lv, W. Lv, and J. Sun, "Convergence dynamics of stochastic reaction-diffusion recurrent neural networks in continuously distributed delays," Nonlinear Analysis: Real World Applications, vol. 9, no. 4, pp. 1590-1606, 2008.

[23] Z. Wang and H. Zhang, "Global asymptotic stability of reaction-diffusion cohen-grossberg neural networks with continuously distributed delays," IEEE Transactions on Neural Networks, vol. 21, no. 1, pp. 39-49, 2010.

[24] Q. Song and Z. Wang, "Dynamical behaviors of fuzzy reaction-diffusion periodic cellular neural networks with variable coefficients and delays," Applied Mathematical Modelling, vol. 33, no. 9, pp. 3533-3545, 2009.

[25] C. Hu, H. Jiang, and Z. Teng, "Impulsive control and synchronization for delayed neural networks with reaction-diffusion terms," IEEE Transactions on Neural Networks, vol. 21, no. 1, pp. 67-81, 2010.

[26] K. Li and Q. Song, "Exponential stability of impulsive Cohen-Grossberg neural networks with timevarying delays and reaction-diffusion terms," Neurocomputing, vol. 72, no. 1-3, pp. 231-240, 2008.

[27] J. Qiu, "Exponential stability of impulsive neural networks with time-varying delays and reactiondiffusion terms," Neurocomputing, vol. 70, no. 4-6, pp. 1102-1108, 2007.

[28] L. Wan and Q. Zhou, "Exponential stability of stochastic reaction-diffusion Cohen-Grossberg neural networks with delays," Applied Mathematics and Computation, vol. 206, no. 2, pp. 818-824, 2008.

[29] X. Xu, J. Zhang, and W. Zhang, "Mean square exponential stability of stochastic neural networks with reaction-diffusion terms and delays," Applied Mathematics Letters, vol. 24, no. 1, pp. 5-11, 2011.

[30] P. Balasubramaniam and C. Vidhya, "Global asymptotic stability of stochastic BAM neural networks with distributed delays and reaction-diffusion terms," Journal of Computational and Applied Mathematics, vol. 234, no. 12, pp. 3458-3466, 2010.

[31] W. Zhang, J. Li, and N. Shi, "Stability analysis for stochastic Markovian jump reaction-diffusion neural networks with partially known transition probabilities and mixed time delays," Discrete Dynamics in Nature and Society, vol. 2012, Article ID 524187, 17 pages, 2012.

[32] W. Zhang and J. Li, "Global exponential synchronization of delayed BAM neural networks with reaction-diffusion terms and the Neumann boundary conditions," Boundary Value Problems, vol. 2012, article 2, 2012. 
[33] W. Y. Zhang and J. M. Li, "Global exponential stability of reaction-diffusion neural networks with discrete and distributed time-varying delays," Chinese Physics B, vol. 20, no. 3, Article ID 030701, 2011.

[34] S. Haykin, Neural Networks, Prentice-Hall, Upper Saddle River, NJ, USA, 1994.

[35] W. Zhu, "Global exponential stability of impulsive reaction-diffusion equation with variable delays," Applied Mathematics and Computation, vol. 205, no. 1, pp. 362-369, 2008.

[36] X.-X. Liao and J. Li, "Stability in Gilpin-Ayala competition models with diffusion," Nonlinear Analysis: Theory, Methods \& Applications, vol. 28, no. 10, pp. 1751-1758, 1997.

[37] V. Lakshmikantham and S. Leela, Differential and Integral Inequalities, Academic, New York, NY, USA, 1969.

[38] J. Liang, Z. Wang, Y. Liu, and X. Liu, "Global synchronization control of general delayed discretetime networks with stochastic coupling and disturbances," IEEE Transactions on Systems, Man, and Cybernetics, Part B, vol. 38, no. 4, pp. 1073-1083, 2008. 


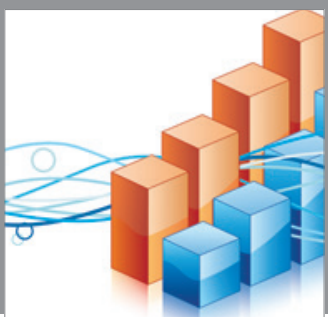

Advances in

Operations Research

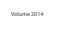

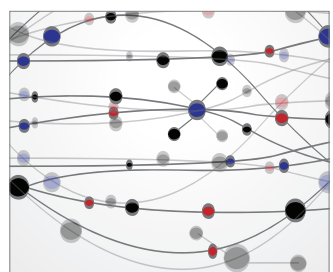

\section{The Scientific} World Journal
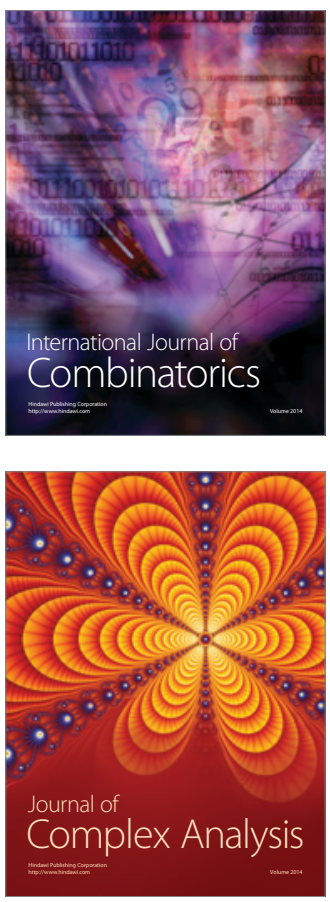

International Journal of

Mathematics and

Mathematical

Sciences
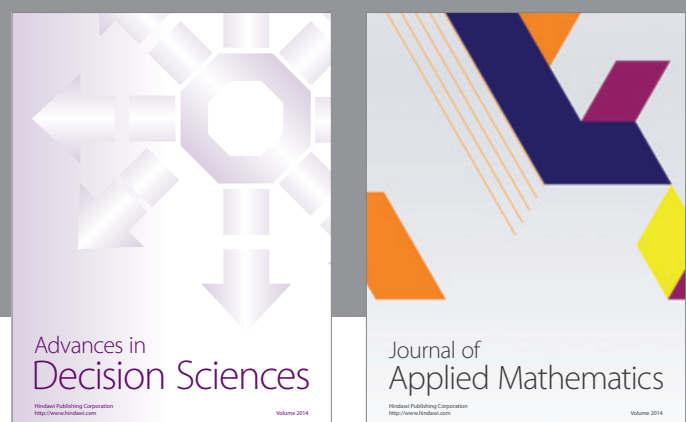

Journal of

Applied Mathematics
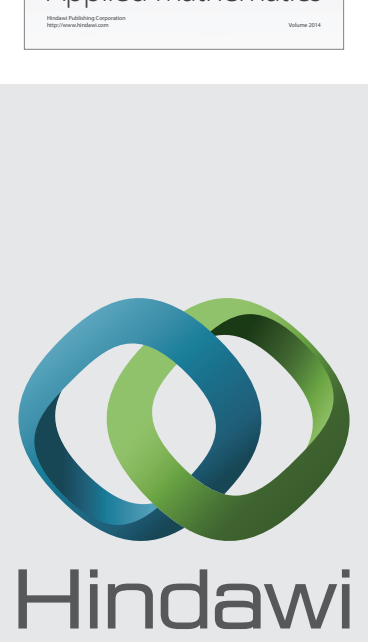

Submit your manuscripts at http://www.hindawi.com
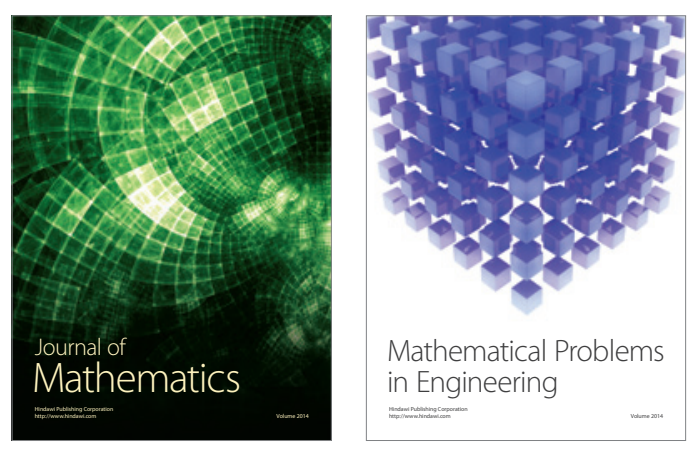

Mathematical Problems in Engineering
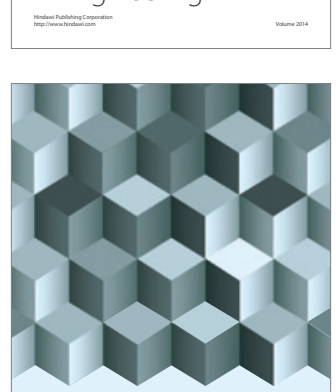

Journal of

Function Spaces
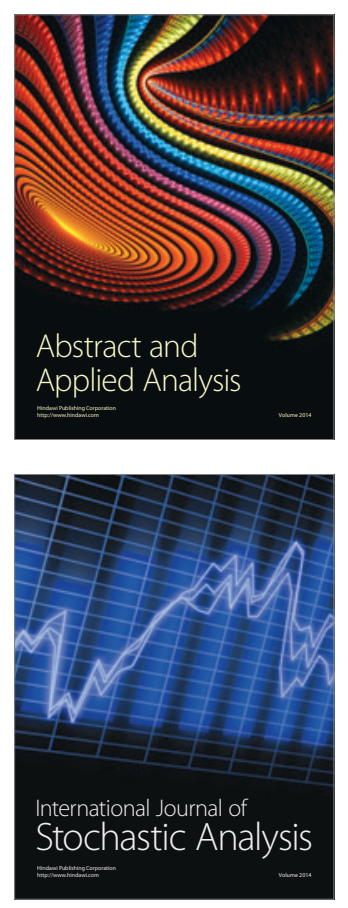

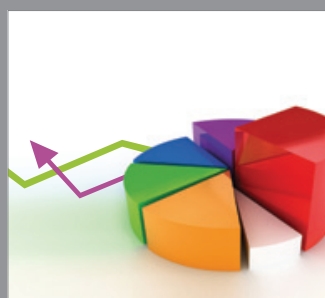

ournal of

Probability and Statistics

Promensencen
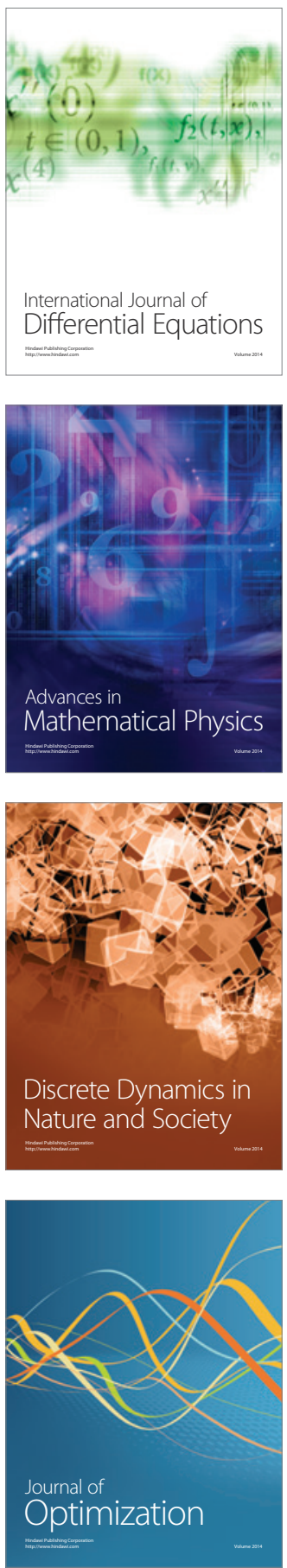